\title{
ATIVIDADES DA VIDA DIÁRIA DE PACIENTES APÓS TRANSPLANTE PULMONAR
}

Susana Beatriz de Souza Pena ${ }^{1}$, Ana Carine Goersch Silva², Isakelly de Oliveira Ramos ${ }^{3}$, Aglauvanir Soares Barbosa ${ }^{4}$ Rita Mônica Borges Studart 5

Objetivo: conhecer as atividades da vida diária através da percepção dos pacientes sobre os cuidados após o transplante pulmonar. Metodologia: estudo descritivo, com abordagem qualitativa, através de grupo focal com 10 pacientes, no periodo de março a abril de 2018. Na análise dos dados foi utilizada a análise do conteúdo. Respeitaram-se os princípios éticos da pesquisa envolvendo seres humanos e o estudo foi aprovado pelo Comitê de Ética com o número 1.146.838. Resultados: emergiram três categorias temáticas: Atividades da vida diária; Cuidados após o transplante pulmonar e Nivel de entendimento. Conclusão: o estudo identificou que os pacientes desenvolveram atividades de autocuidado como tomar banho sozinho e comer sem ajuda, modificaram hábitos alimentares, e aumentaram os cuidados com as medicações prescritas. .

Descritores: Transplante de Pulmão, Autocuidado, Enfermagem.

\section{DAILY LIFE ACTIVITIES OF PATIENTS AFTER LUNG TRANSPLANTATION}

Objective: To know the activities of daily living through the perception of patients about care after lung transplantation. Methodolgy: a descriptive study with a qualitative approach through a focus group with 10 patients, from March to April 2018. In the data analysis was used the content analysis. The ethical principles of research involving human subjects were respected and the study was approved by the Ethics Committee under number 1.146.838. Results: three thematic categories emerged: instrumental activities of daily living; Care after lung transplantation and Level of understanding. Conclusion: The study found that patients developed self-care activities such as bathing alone and eating unaided, modified eating habits, and increased care with prescribed medications.

Descriptors: Lung Transplantation, Self Care, Nursing.

\section{ACTIVIDADES DIARIAS DE LA VIDA DE LOS PACIENTES DESPUÉS DEL TRASPLANTE DE PULMÓN}

Objetivo: Conocer lãs actividades de la vida diária através de La percepción de los pacientes sobre la atención después del trasplante pulmonar. Metodología: um estúdio descriptivo com um enfoque cualitativo através de um grupo focal com 10 pacientes, de marzo a abril de 2018. En el análisis de datos se utilizo el análisis de contenido. Se respetaron los princípios éticos de la investigación en seres humanos y el estúdio fue aprobado por el Comitê de Ética con el número 1.146.838. Resultados: surgieron tres categorias temáticas: actividades de la vida diária; Atención después del trasplante de pulmón y nível de comprensión. Conclusión: el estúdio encontro que los pacientes desarrollaron actividades de autocuidado como bañarse solo y comer sin ayuda, hábitos alimentícios modificados y uma mayor atención con medicamentos recetados.

Descriptores: Trasplante de Pulmón, Autocuidado, Enfermería.

Enfermeira. Mestranda do Mestrado Profissional em Tecnologia e Inovação em Enfermagem, acordo COFEN, Universidade de Fortaleza (Unifor). Fortaleza, Ce. Enfermeira. Mestranda do Mestrado Profissional em Tecnologia e Inovação em Enfermagem, acordo COFEN, Universidade de Fortaleza (Unifor). Fortaleza, Ce. Enfermeira. Mestranda do Mestrado Profissional em Tecnologia e Inovação em Enfermagem, acordo COFEN, Universidade de Fortaleza (Unifor). Fortaleza, Ce. Enfermeira. Mestranda em Enfermagem, Universidade da Integração Internacional da Lusofonia Afro-Brasileira (Unilab). Redenção, Ce.

Doutora em Enfermagem. Docente do Mestrado profissional em Enfermagem, Universidade de Fortaleza (Unifor), Fortaleza, Ce.Autor correspondente: Aglauvanir Soares Barbosa. E-mail: glauasbl@gmail.com 


\section{INTRODUÇÃO}

O transplante pulmonar não significa cura, mas a possibilidade de uma nova perspectiva de vida frente ao tratamento. O paciente conta com assistência contínua de uma equipe multiprofissional que prescreverá toda terapêutica a fim de reduzir os riscos de rejeição, porém para tal sucesso é primordial que esse transplantado tenha adesão às orientações rigorosas estabelecidas pelos profissionais de saúde ${ }^{(1,2)}$

No Brasil, de acordo com dados do Registro Brasileiro de Transplantes (2019), foram contabilizados 1.180 transplantes absolutos de pulmão, com registros que vão desde 1997. Até março deste ano, haviam 177 pacientes ativos em lista de espera, para um trimestre que notificou 27 transplantes pulmonares no país, com seis equipes ativas em três estados: Ceará, Rio Grande do Sul e São Paulo.

De acordo com dados internacionais, as taxas de sobrevida apontam números elevados no primeiro ano, entre 75 e $85 \%$. Porém, no Brasil, essa sobrevida é reduzida para 68\%(3.4). Esse aspecto de sobrevida tem relação com aumento de doadores, melhora do desempenho funcional precoce, diagnóstico mais efetivo das infecções do pós-operatório imediato e tardio, manejo das complicações relacionadas com rejeição crônica, sobretudo a adesão ao tratamento(5)

Sendo assim, a alta hospitalar significa um momento de grande felicidade para os pacientes e familiares, mas ao mesmo tempo um momento de medo e insegurança por não ter mais a equipe de saúde ao seu lado. Isso gera uma demanda de informações a respeito do procedimento e dos cuidados necessários, que poderão se perder ao longo dos dias, sendo importante receber orientações sobre os cuidados necessários na sua rotina diária.

Nesse contexto, as atividades da vida diária são resultados esperados, conforme o Nursing Outcomes Classification (NOC), associados ao diagnóstico de enfermagem da North American Nursing Diagnosis Association (NANDA) onde o autocuidado é objeto desse trabalho na ótica da percepção dos pacientes sobre os cuidados após o transplante pulmonar. Além disso, são instrumentos notórios e que qualificam a consulta de enfermagem, conforme Resolução COFEN no 358/2009.

Logo o êxito no transplante depende da participação do paciente no processo de autocuidado, ou seja, no engajamento ao seu tratamento contínuo ${ }^{(6,7)}$. O autocuidado é uma função reguladora que permite às pessoas desempenharem atividades que visem à preservação da vida, da saúde, do desenvolvimento e do bem-estar ${ }^{(8)}$. Além disso, o autocuidado é entendido como resultado do cuidado de enfermagem, visto como orientação subjacente à atividade do enfermeiro.

Nesse âmbito, autocuidado está associado a autonomia, independência e responsabilidade pessoal. É um fenômeno complexo e multidimensional, conceituado por um processo de saúde e bem-estar dos indivíduos, na perspectiva da capacidade de tomada de iniciativa, responsabilidade e funcionarem de forma eficaz no desenvolvimento do seu potencial para a saúde ${ }^{(9)}$.

Ademais adesão ganha conotação e importância quando se complementa com o sentido do autocuidado. O significado da adesão é algo particular, próprio de cada paciente, que pressupõe o livre direito do paciente escolher em adotar ou não determinada recomendação médica ${ }^{(5)}$. Portanto, toda a equipe multidisciplinar que assiste o transplantado, precisa conhecer a singularidade deles, considerando sua história, personalidade e visão do paciente em querer aderir a um novo estilo de vida.

Portanto o estudo será de grande relevância para que os profissionais de saúde, em especial enfermeiros, possam promover a saúde do paciente transplantado orientando sobre práticas de autocuidado e importância da adesão ao tratamento. Servirá também de subsídios e aporte para a comunidade científica com o intuito de ampliar conhecimentos.

\section{OBJETIVO}

O objetivo do estudo foi conhecer as atividades da vida diária mediante a percepção dos pacientes sobre os cuidados após o transplante pulmonar.

\section{METODOLOGIA}

\section{Tipo de estudo}

Trata-se de um estudo descritivo, com abordagem qualitativa.

\section{Participantes da pesquisa}

Participaram da pesquisa 10 pacientes transplantados que estavam em acompanhamento junto à equipe do ambulatório, de forma intencional, sendo escolhidos em conjunto com o serviço de saúde, considerando-se a disponibilidade dos sujeitos em participar da investigação. Salienta-se que todas as entrevistas realizadas na coleta de informações foram utilizadas na análise.

Os critérios de inclusão foram: ser transplantado pulmonar; estar em acompanhamento ambulatorial; não apresentar déficit cognitivo e ter condições de estabelecer diálogo.

\section{Local do estudo}

Este estudo foi realizado no serviço de seguimento ambulatorial do transplante pulmonar, de um hospital 
terciário especializado no diagnóstico e tratamento de doenças cardiacas e pulmonares.

\section{Coleta de dados}

Utilizou-se como uma das etapas da coleta de dados o grupo focal, técnica na qual a interação é parte integrante, pois permite que os participantes explorem seus pontos de vista sobre determinado assunto e gera perguntas, dúvidas ou respostas em um período não superior a duas horas ${ }^{(10)}$.

Os participantes do grupo focal foram de seis a dez e possuiam, entre si, ao menos uma característica comum, constituindo uma amostra intencional. Para as discussões fezse necessária a participação de um pesquisador/moderador, que iniciou a temática principal estimulando a interação dos participantes.

A primeira sessão foi realizada com seis pacientes e a segunda com quatro, em razão da abstinência e ausência de pacientes agendados para a consulta. Importante destacar que as cadeiras foram organizadas em círculos com o objetivo de promover o contato visual e favorecer a visão e interação entre eles ${ }^{(10)}$.

Após a apresentação dos objetivos da pesquisa, foi utilizado um roteiro com as seguintes questões focais: Quais as atividades da sua vida diária? Quais são os cuidados que você gostaria de saber depois de ter realizado o transplante? O que você entende sobre seu transplante? O tempo de duração dos grupos focais variou entre 40 minutos e uma hora.

\section{Procedimentos de análise dos dados}

Os depoimentos foram gravados, transcritos e organizados em torno dos manejos e cuidados no pósoperatório mediato. Para a análise dos dados foi utilizada a técnica de análise do conteúdo, que busca os sentidos encontrados nos documentos, notas de observação de diários de campo, entrevistas gravadas ${ }^{(11)}$, permitindo a identificação das categorias temáticas por meio dos depoimentos apresentados pelos sujeitos do estudo e assim facilitando a compreensão do problema em questão.

\section{Procedimentos éticos}

Foram respeitados os princípios éticos e legais da pesquisa envolvendo seres humanos preconizados pela Resolução 466/2012 e o estudo recebeu parecer favorável pelo Comitê de Ética com o número 1.146.838.

\section{RESULTADOS}

$\mathrm{Na}$ apresentação das falas, os participantes foram codificados para garantir seu anonimato com a letra "E" de entrevistado e um número dado a cada sujeito de 1 a 10. A composição do grupo apresentou a seguinte configuração: média de idade de 32,5 anos, com predominância do sexo masculino.

A análise das falas revelou as seguintes categorias temáticas: Atividades da vida diária; Cuidados após o transplante pulmonar e Nivel de entendimento.

\section{Atividades da vida diária}

Ao longo do conhecimento empreendido das atividades de vida dos pacientes submetidos ao transplante de pulmão, percebeu-se uma grande mudança, evidenciando novos condicionamentos que corroboraram em sensações positivas na qualidade de vida dos transplantados, como é expresso nas falas a seguir:

[...]tudo diferente, desde voltar a aprender a respirar, aprender a andar, aprender a falar, aprender a comer, aprender a lembrar das pessoas [...] então tudo foi como se fosse um recém-nascido mesmo (E2).

Antes era de cadeira de roda, com oxigênio direto, 24 horas no oxigênio com 6 litros, era bem debilitado, muita dor no corpo, não conseguia fazer nada [...] então minha atividade diária mudou muito, hoje tem que ter outros cuidados (E4).

Antes do transplante eu parei até de estudar, não conseguia comer quase nada, até pra dormir tinha que ser com oxigênio. Era 24 horas. Minhas atividades depois do transplante estão bem diferentes, mas continuo tendo que ter muito cuidado (E5).

A coisa que eu fiz mais festa quando me transplantei, foi para tomar um banho, porque eu tomava banho com uma cadeira ligada na bala de oxigênio [...] em poucos dias (após a cirurgia), eu já estava reabilitada (E10).

O recondicionamento alimentar torna-se fator importante para o paciente, pois além de reduzir possiveis infecções, evita sobrepeso ou baixo peso, bem como doenças associadas à nutrição e metabolismo. Como depreende-se nas falas abaixo:

Minha rotina que mudou mais foi que tive que mudar minha alimentação toda. Camarão eu tive que tirar e refrigerante que eu era viciada (E3).

A alimentação tem sido de acordo com o que a nutricionista passa. Comida sem sal, controle no açúcar. As vezes sinto vontade de comer algo que não deve, algum doce, mas eu tento evitar (E6).

Não andar no sol, pegar poeira, essas coisas [...] me 
alimentar direito que aqui, aculá, vacilo [...] e as vezes como carne de porco, perigoso! depois eu penso: será que faz mal? eu fico pensando, valha me Deus (E7).

\section{Cuidados após o transplante pulmonar}

A sensibilização do autocuidado é algo desafiante para o paciente, pois o mesmo precisa compreender os riscos, a fim de executar as estratégias de cuidado prescritas. Como podem ser vistos a seguir:

[...]não me importava com alimentação, não me importava com o fato de ter tapetes, cortinas, antigamente, eu tinha tudo [...] a gente passa a se policiar mais (E2).

O meu cuidado agora é onde tem muitas pessoas não posso ficar, né? [...]onde tem muita poeira, fumaça ... não posso ficar. Gente que tão fumando isso ai é um veneno pra $\operatorname{mim}(E 1)$.

Um fato que se tornou complicado foi o meu trabalho, eu sempre trabalhei e agora pra mim voltar pra minha área não posso volta para minha área que é recursos humanos porque é muito contato com pessoas e documentos (E3).

Tem uma tabelinha em casa na parede, com os remédios embaixo e também uso os alarmes dos telefones. Acordo cedo, mesmo sem querer, tomo os remédios $e$ volto a dormir (E5).

Eu tenho muito cuidado com as medicações, com essas coisas [...] eu gosto muito de comer, mas eu não como nada que eu tenha costume sem perguntar pra elas (E6).

A enfermagem tem papel fundamental em levantar mecanismos de motivação ao seu paciente transplantado, pois são muitas as restrições, conforme é ratificado nas falas:

O cuidado com horário da alimentação e da medicação é prioritário [...] se você tiver esse controle, dá pra levar [...] agora quem não tem esses horários reservados, ai é dificil (E7).

Depois do transplante a higiene dos alimentos é muito séria, a princípio, quando eu

[...]os cuidados é que eu não posso mais mexer com poeira, tomar banho com água quente do sol ne? [...] fumaça, eu não posso também [...] se for pra sentar num banco quente, eu prefiro ficar em pé (ElO).

\section{Nivel de entendimento}

Essa categoria busca explorar o entendimento dos pacientes sobre seu cotidiano e as mudanças presentes no dia-a-dia, associadas ao tratamento, além das principais dificuldades decorrentes frente à realização de um transplante pulmonar.

Percebem-se nos diálogos seguintes a falta de conhecimento dos pacientes a respeito do transplante, tanto antes, durante e após a cirurgia. Bem como sobre os estilos de vida que precisarão seguir para conduzir o novo órgão:

Então quando sai, sempre uso a máscara, né? É isso ai que é minha orientação. Ter muito cuidado com a minha vida agora. Daqui pra frente. Não sabia nada disso (El). Eu pesquisava muito antes de transplantar, então muitas coisas que eles falaram, eu já sabia, mas quando eu acordei entubada, foi a pior parte de todas, essa parte eu não sabia de nada (E4).

Não sabia nada dessa parte de facilidade de ter infecção. A última tomografia que fiz tive que esperar muito porque para desinfetar a máquina porque teve um paciente infectado que fez antes de $\operatorname{mim}(E 9)$.

Dentre as várias estratégias vigentes no processo instrutivo, verifica-se que os materiais educativos assumem importante papel na promoção da saúde, pois promovem a mediação de conteúdos de aprendizagem e funcionam como recurso disponível, para que o paciente e sua família possam compreender, refletir, além de consultar as recomendações prescritas pela equipe de saúde ${ }^{(6)}$.

Eu acho que deveria ter mais investimento do governo, pra ser mais divulgado o transplante. Já vai fazer muito tempo que ninguém é transplantado. O pessoal tá piorando bastante e desconhece esse tipo de tratamento (E8).

Essa condição de cronicidade é observada nos recortes abaixo, sobre aspectos do novo cotidiano do transplantado de pulmão:

Os medicamentos que devem ser tomados nos horários certos. Tudo isso você tem que entender [...] eu tinha habito de acordar na hora do almoço, mas hoje em dia acordo as seis horas por conta dos medicamentos, E6). Temos que entender pra começar a tomar os medicamentos. Eu uso um despertador do celular, sempre. tento estar com o celular na mão. coloco o nome do medicamento e o horário (E7).

As taxas de sobrevida do transplantado têm influência direta com seu autocuidado. Esse aspecto é rotineiramente abordado em toda trajetória do transplantado. Nas consultas ambulatoriais, a equipe de saúde utiliza técnicas 
de encorajamento, continuamente, em prol da adaptação ao novo estilo de vida que deve ser adquirido(7)

Eu acho muito difícil, tem que ter mais cuidado com tudo, por onde você anda, com quem você tá, o que você vai comer, os horários de medicamentos, a hora que vai dormir e acordar... cansa (E2)

Eu tive que aprender todas as medicações, mas no começo não foi fácil [...]Eu moro só, tenho uma filha que mora perto, mas trabalha e não posso contar com ela (E10).

O processo educativo é um instrumento de socialização de conhecimentos, de promoção da saúde e de prevenção de doenças, pois contribui na valorização da autonomia, da autoestima, da autoconfiança e realização, sendo capaz de transformar posturas e atitudes.

É uma função essencial à prática do enfermeiro, não somente por meio da comunicação de conteúdo, mas também, no desenvolvimento e avaliação dos recursos educativos produzidos nos seus clientes para o autocuidado(15).

\section{DISCUSSÃO}

O transplante pulmonar é uma cirurgia que não significa a cura, mas a possibilidade de uma nova perspectiva de vida ao tratamento. O paciente submetido a tal intervenção conta com assistência contínua de uma equipe multiprofissional que prescreverá toda a terapêutica a fim de reduzir os riscos de rejeição, porém para tal sucesso é primordial que esse transplantado tenha adesão às orientações rigorosas estabelecidas pelos profissionais de saúde ${ }^{(12)}$.

Otratamento após o transplanteapresenta características de uma condição crônica, de uma patologia permanente que necessita de um acompanhamento especializado contínuo. O que implica em tratamento ininterrupto com regime terapêutico complexo prescrito por uma equipe multidisciplinar em diferentes âmbitos, como: restrições nutricionais, limitações de horários das refeições, abstinência de bebidas alcoólicas e tabagismo, sexo seguro, terapia medicamentosa adequada, exames periódicos permanentes, além de restrições à socialização(5).

Surge como a única alternativa de sobrevivência do paciente acometido por uma doença pulmonar avançada, na qual já foram esgotadas todas terapêuticas médicas existentes. Essas doenças pulmonares, caracterizam-se por um grupo heterogêneo de enfermidades que possuem letalidade elevada, a despeito do considerável progresso nos tratamentos farmacológicos disponíveis ${ }^{(13)}$.

Para o êxito do transplante, se faz necessário o entendimento do pacientearespeito daprática deautocuidado, e que o mesmo se responsabilize e reconheça a importância de sua participação nesse processo. $O$ transplantado requer assistência específica e de qualidade, exigindo do profissional enfermeiro o constante acompanhamento das necessidades do paciente, além de permanente estímulo e motivação à prática do autocuidado(7).

O paciente submetido ao transplante pulmonar acaba, ao longo do tempo, perdendo a motivação na contínua adesão, e esse descumprimento para alguns autores ${ }^{(5)}$, não pode ser entendido como desobediência. A relação entre profissionais de saúde, familiares e pacientes deve atingir a coesão, e isso pode ser atingido através do treinamento na habilidade da equipe em comunicar-se, resultando em taxas de adesão significativamente maiores ${ }^{(5)}$.

Dentro das funções que o enfermeiro, ao longo de seu percurso acadêmico e profissional, aprender a ter é a responsabilidade do cuidar do paciente e de ensinar o autocuidado, promovendo e recuperando a saúde. Sendo assim, uma das preocupações que se deve ter é educar o paciente a se cuidar, provendo e gerenciando o autocuidado em sua rotina, buscando promoção da saúde, recuperandose de doenças ou injúrias, convivendo com seus efeitos ${ }^{(14)}$.

A falta de conhecimento contínuo e constante pelos pacientes podem leva-los a condições de riscos e possiveis rejeições, afetando o enxerto. Há também a constatação de que apesar de todo sacrifício não existe a possibilidade de cura após um transplante permanecendo a necessidade do contato com a equipe de saúde e o comprometimento com as medicações, assim como as limitações tanto alimentares como as que exigem um cuidado corporal $(15,16)$.

O comprometimento no autocuidado acontece quando o paciente se vê atuante no seu processo de saúde-doença, ou seja, quando há o empoderamento no seu estilo de vida(8). Sendo imprescindivel uma equipe de saúde comprometida com o cuidado aos seus pacientes, onde todas as orientações possam ser repassadas da forma mais simples possivel, de maneira que o paciente saiba seus riscos e benefícios, bem como os cuidados necessários para uma melhor sobrevida com o enxerto.

\section{Limitações do estudo}

A limitação deste estudo refere-se ao desafio da pesquisa de entrevistar pacientes após o transplante, um procedimento delicado, precisando de manejo adequado do profissional da saúde para abordagem eficaz, e ainda um grupo pequeno e apenas de um centro de transplantes.

\section{Contribuições do estudo para a prática}

A contribuição desta pesquisa é de explicitar as atividades desenvolvidas sob o relato dos pacientes, sendo de extrema 
relevância para fomentar novas publicações relacionadas à aos transplantados.

\section{CONCLUSÃO}

O estudo identificou que os pacientes desenvolveram atividades de autocuidado como tomar banho sozinho e comer sem ajuda, modificaram hábitos alimentares, e aumentaram os cuidados com as medicações prescritas. Nesse contexto, mediante a percepção dos pacientes sobre os cuidados após o transplante pulmonar, evidenciou através das falas dos pacientes que o tratamento é complexo e exige compromisso de todos os envolvidos.
Nessa perspectiva, percebe-se nas falas dos pacientes que a rotina diária é cansativa e ao longo dos anos torna-se cansativa favorecendo a má adesão do tratamento. Sendo assim sugere-se que as técnicas e decisões não devem ser impostas pela equipe interdisciplinar, sendo preciso conhecer a singularidade, as características e dificuldades de cada pessoa em aderir o tratamento complexo do transplante.

\section{Contribuições dos autores}

Concepção e/ou desenho: Pena SB de S e Studart RMB; Redação, Análise e interpretação dos dados: Pena SB de S; Studart RMB; Barbosa AS; SILVAACG e Ramos I de O; Revisão crítica: Pena SB de S; Studart RMB e Barbosa AS.

\section{REFERÊNCIAS}

1. Gomes Neto A, Monteiro Nogueira AS, Lopes De Medeiros I, Fernandes Viana De Araujo R, Carvalho Santos R, Sampaio Viana CM, et al. Singleand Double-Lung Transplantation: Results of an Initial Experience of 39 Cases in Ceará (Northeast Brazil). Transplantation Proceedings [internet]. 2018 [cited 2019 ago 16];50(3):815-8. Available from:

2. https://www.ncbi.nlm.nih.gov/pubmed/296614442. Marinho BBO Santos ATF, Figueredo AS, Cortez LSAB, Viana MCA, Santos GM, et al. Challenges of Organ Donation: Potential Donors for Transplantation in an Area of Brazil's Northeast. Transplantation Proceedings [internet]. 2018 [cited 2019 ago 16]:50(3):698-701. Available from:

https://www.ncbi.nlm.nih.gov/pubmed/29661419

3. Camargo PCLB, Teixeira RHO, Carraro RM, Campos SV, Afonso Junior JE, Costa AN, Fernandes LM, Abdalla LG, Samano MN, Fernandes PMP. Transplante pulmonar: abordagem geral sobre seus principais aspectos. J Bras Pneumol. [internet]. 2015 [cited 2019 ago 16]:41(6):547-553. Available from: http://www.jornaldepneumologia.com.br/detalhe_artigo.as$p ? i d=2464$

4. RBT. Registro Brasileiro de Transplantes. Dimensionamento dos Transplantes no Brasil e em cada estado (2010-2017). Veículo oficial da Associação Brasileira de Transplante de Órgãos (ABTO)[internet]. 2017 [cited 2019 ago 16];Ano XXIII:4. . Available from:

http://www.abto.org.br/abtov03/Upload/file/RBT/2017/rbt-imprensa-leitura-compressed.pdf

5. Costa AN, Hojaij EM, Mello LS de, Melo FX de, Camargo PCLB de, Campos SV, et al. Falta de adesão ao tratamento em pacientes submetidos a transplante pulmonar: uma questão de vida ou morte. Jornal Brasileiro de Pneumologia [internet]. 2015 [cited 2019 ago 16]:41(1):95-7. Available from: http://www.jornaldepneumologia.com.br/detalhe_artigo.asp?id $=2376$

6. Moura IH de, Silva AFR da, Rocha A do ES de H, Lima LH de O, Moreira TMM, Silva ARV da, et al. Construção e validação de material educativo para prevenção de síndrome metabólica em adolescentes. Revista Latino-Americana de Enfermagem [Internet]. 2017 [cited jun 20];25. Available from:

http://www.scielo.br/scielo.php?script=sci_arttext\&pi$d=$ do104-11692017000100383\&lng=en\&nrm=iso\&tlng=pt

7. Custódio IL. Avaliação do autocuidado de pacientes após transplante cardiaco acompanhado na consulta de enfermagem. Dissertação de mestrado. Mestrado em enfermagem na promoção da saúde. Universidade Federal do Ceará [internet]. 2012. [cited 2019 ago 16]. Available from: http://www.repositorio.ufc.br/handle/riufc/3993
8. Galvão MT dos RLS, Janeiro JM da SV. O autocuidado em enfermagem: autogestão, automonitorização e gestão sintomática como conceitos relacionados. Revista Mineira de Enfermagem [internet]. 2013 [cited 2019 ago 16]:17(1):226-36. Available from: http://www.reme.org.br/ artigo/detalhes/593

9. Queirós PJP, Vidinha TS dos S, Almeida Filho AJ de. Autocuidado: o contributo teórico de Orem para a disciplina e profissão de Enfermagem. Revista de Enfermagem Referência [internet]. 2014 [cited ago 16]:serieIV:3. Available from: http://www.scielo.mec.pt/pdf/ref/vserIVn3/serIVn3al8.pdf

10. Backes DS, Colomé JS, Erdmann RH, Lunardi VL. Grupo focal como técnica de coleta e análise de dados em pesquisas qualitativas. O Mundo da Saúde [internet]. 2011 [cited 2019 ago 16]:35(4):438-42. Available from: http://bvsms.saude.gov.br/bvs/artigos/grupo_focal_como_tecnica_coleta_analise_dados_pesquisa_qualitativa.pdf

11. Alexandre NMC, Coluci MZO. Validade de conteúdo nos processos de construção e adaptação de instrumentos de medidas. Ciência Eamp: Saúde Coletiva [internet].2011 [cited 2019 ago 16]:16(7):3061-8. Available from: http://www.scielo.br/scielo.php?script=sci_arttext\&pi$d=S 1413-81232011000800006$

12. Ionta MR. Análise do perfil clínico e epidemiológico dos pacientes que realizaram transplante renal em um hospital beneficente. Revista Paraense de Medicina [internet]. 2013 [cited 2019 ago 16]:5. Available from: http://files.bvs.br/upload/S/0101-5907/2013/v27n4/a4080.pdf

13. Mariani AW, Pêgo-Fernandes PM, Abdalla LG, Jatene FB. Recondicionamento pulmonar ex vivo: uma nova era para o transplante pulmonar. Jornal Brasileiro de Pneumologia [internet]. 2012 [cited 2019 ago 16];38(6):776-85. Available from: http://www.scielo.br/scielo.php?scrip$\mathrm{t}=$ sci_arttextEpid=S1806-37132012000600015

14. Orem DE, Taylor SG. Reflections on Nursing Practice Science The Nature, the Structure, and the Foundation of Nursing Sciences. Nursing science quarterly [internet] 2011 [cited 2019 ago 16];24(1):35-41. Available from: https://www.ncbi.nlm.nih.gov/pubmed/21220574

15. Oliveira SC de, Lopes MV de O, Fernandes AFC. Development and validation of an educational booklet for healthy eating during pregnancy. Revista Latino-Americana de Enfermagem [internet]. 2014 [cited 2019 ago 16];22(4):611-20. Available from: http://www.scielo.br/pdf/rlae/ v22n4/pt_0104-1169-rlae-22-04-00611.pdf

16. Afonso Júnior JE, Werebe E de C, Carraro RM, Teixeira RH de OB, Fernandes LM, Abdalla LG, et al. Lung transplantation. Einstein (São Paulo) [internet]. 2015 [cited 2019 ago 16];13(2):297-304. Available from: http:// www.scielo.br/pdf/eins/v13n2/pt_1679-4508-eins-13-2-0297.pdf 